\title{
Single balloon aberration into the uterus despite no rupture of the fetal membranes: a case report
}

\begin{abstract}
An obstetrical single balloon is used to facilitate ripening of the cervix and is widely used because it is relatively safe.

Even when used that tool correctly, sometimes unexpected events might occur leaving us puzzled. We report a case in which a single balloon catheter aberrated fully into the uterus without rupture of the membranes at 41 weeks of gestation. Keeping in mind the possibility of placental abruption, artificial rupture of the membranes was performed, which triggered uterine contractions and, finally, resulted in a natural delivery.

While the need for a single balloon to be inserted by a skilled practitioner and confirmation of the position of the balloon are essential, in the present case, we were keenly aware of the need for flexibility in our resourcefulness in an unexpected situation. If a single balloon enters into the uterus completely without rapture of the membranes, an amniotomy is suggested.
\end{abstract}

Keywords: Obstetrical single balloon, Delivery, Artificial rupture of the membranes
Volume 8 Issue 3 - 2017

\author{
Atsushi Yanaihara, Shirei Ougi, Kenichirou \\ Motomura, Aguri Hirano, Shin Takenaka, \\ Takumi Yanaihara \\ Yanaihara Clinic for Women, Japan
}

Correspondence: Atsushi Yanaihara MD, Ph.D, Yanaihara Clinic for Women, 2-17-18 Ofuna Kamakura Kanagawa Japan Zip2470056, Tel 8|467500 I I2, Fax 8|467500 I 3,

Email at123y@yahoo.co.jp

Received: October 25, 2017 | Published: December 29, 2017

\section{Introduction}

Cervical ripening is as important part of the process of labor induction. While other mechanical methods are available, such as extra-amniotic saline infusion ${ }^{1}$ and the use of osmotic dilators and double-balloon catheters, an obstetrical single balloon is commonly used to facilitate ripening of the cervix. ${ }^{2}$ It is widely used because it is relatively safe and has few adverse effects. Single balloon catheter, which is commonly used in this country, is an improvised version of a Foley's catheter. This catheter is used in approximately 40,000 cases a year in this country. The single balloon catheter is inexpensive and easy to use because the neck of the catheter is thick compared to that of a Foley's catheter.

We report a rare case of single balloon catheter aberration into the uterus without rupture of the membranes at 41 weeks of gestation wherein artificial rupture of the membranes was performed, which triggered uterine contractions and, finally, resulted in a natural delivery.

\section{Case presentation}

The patient was a 32-year-old lady, gravida 1 and para 1 , who was 41 weeks and 2 days into pregnancy. She was admitted to the hospital one day prior for labor induction after her due date had passed. On admission, she was found to have $1 \mathrm{~cm}$ of cervical os with $30 \%$ effacement of the cervix and the fetus at station -3 without rupture of the membranes. A single balloon, metreurynter (MINI METRO ${ }^{\circledR}$, Soft medical Co., Ltd., Tokyo, Japan), was injected with $40 \mathrm{ml}$ of distilled water and inserted into the cervical canal for cervical ripening.

The practitioner determined that the insertion was shallow (not within the internal OS) and re-inserted the metreurynter. Cardiotocography (CTG) was performed prior to the insertion. The variable decelerations were noted 48 minutes after the insertion and a subsequent pelvic examination revealed no change in the pelvic findings and the metreurynter was not palpable. Because the patient had no history or signs of metreurynter prolapse, transabdominal ultrasound was performed, which revealed a round, hypoechoic region
$4 \mathrm{~cm}$ in diameter above the fetal head near the placenta (Figure 1). The patient was, thus, diagnosed with metreurynter aberration. Keeping in mind the possibility of placental abruption, artificial rupture of membranes was performed with a double setup of caesarian section, which triggered uterine contractions. Frequent pelvic examinations were performed and the cervix eventually dilated, resulting in vaginal delivery. After delivery of the fetal head, the metreurynter prolapsed just as the fetal body was delivered. A female baby weighing 3,420 g (APGAR score was 9 at both $1 \mathrm{~min}$ and $5 \mathrm{~min}$ ) was delivered after 1 hour 20 minutes of labor and the postpartum blood loss was 784 $\mathrm{ml}$. Because the delivery was fast, the patient was at risk for atonic bleeding; however, both the mother and child were discharged following an uneventful postpartum course. No problems were noted with the placenta and the location of the metreurynter could not be identified from the fetal membrane opening. This report has been prepared with the approval of the Ethics Committee of Yanaihara Clinic for Women and the informed consent of the patient for publication $(\mathrm{ERBY} / 2,2016)$.

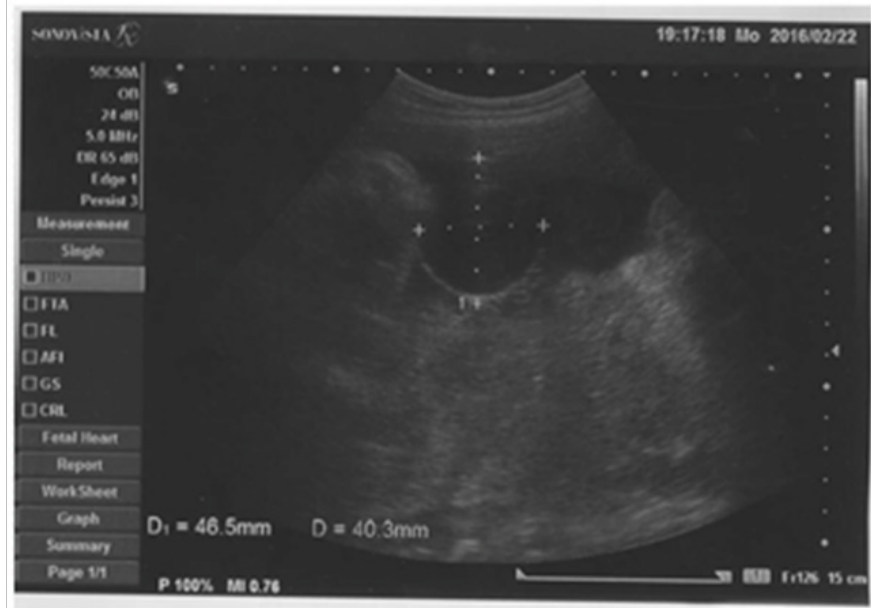

Figure I Ultrasound of obstetrical balloon aberrated into the uterus. 


\section{Discussion}

Inducing labor based on medical indications is a common practice. Furthermore, it is no longer rare to have a planned delivery such as painless delivery. The rate of cesarean sections is high in cases of cervical immaturity, ${ }^{3,4}$ and ripening of the cervix has been reported as an important factor influencing the success of labor induction. ${ }^{3,4}$ The obstetrical single balloons currently being used at most of the facilities in this country are made of silicone and can be used safely without fear of latex allergy.

In Japan, to the best of our knowledge, only three cases of metreurynter aberration into the uterus have been presented at scientific meetings. In all these cases, the aberration occurred after the rupture of fetal membranes (this information is from the manufacturer, unpublished data). This is the first case report to describe the complete aberration of an obstetrical single balloon into the uterus with intact fetal membranes.

When the fetal head has not entered the pelvis, there is a possibility of umbilical cord prolapse or aberration of an obstetrical single balloon between the fetal head and maternal pelvis. ${ }^{5-7}$ This phenomenon is possible if the fetal membranes have ruptured, but an aberration is unexpected with intact membranes. It is likely that the obstetrical single balloon slipped under the fetal membranes when it was pushed strongly and was inflated once it had reached beyond the fetal head. Possible reasons for such an aberration with intact membranes include the vulnerability of the mature fetal membranes and the patient's parity index. In this case, the obstetrical single balloon moved with the fetal movements from its aberrated location until it reached just below the placenta, where it stayed.

Another reason may be the characteristic of the catheters. The lower part of the balloon is shorter than that of a Foley's catheter. When a balloon completely invaginates into the uterus, the water in the balloon cannot be extracted. CTG findings of variable decelerations appear unrelated at first glance, but the possibility that the aberration of the obstetrical single balloon into the uterus was the trigger cannot be ruled out. The biggest focus in this case was the possibility of a mechanical stimulus causing bleeding and placental abruption. There are some reports that placenta abruption occurred after insertion of intrauterine catheters. ${ }^{3,8}$

Therefore, we attempted artificial rupture of the fetal membranes to prevent the progression of membrane abruption, thereby reducing the intrauterine pressure. Rupturing the membranes was expected to forestall placental abruption. We also attempted to facilitate the progress of labor by inducing uterine contractions.

An earlier report described the use of an obstetrical single balloon for cervical ripening in a case of premature rupture of the membranes, where aberration of the obstetrical single balloon was confirmed on pelvic examination the following day. Labor induction was commenced with the expectation that the obstetrical single balloon would be naturally expelled, but a pelvic examination halfway through the course of labor revealed that a part of the obstetrical single balloon was palpable. The obstetrical single balloon was removed with gentle pulling, causing a prolapse of the umbilical cord and resulting in an emergency cesarean section. In two other cases, the MINI METRO was expelled as the fetus was delivered.

Thus, while it is likely that more cases of aberration following the rupture of membranes occur than are reported, to the best of our knowledge, this case report is the first to describe obstetrical single balloon aberration into the uterus despite intact fetal membranes.
Use of transcervical Foley's catheters for cervical ripening is not associated with an increased risk of infectious morbidity. ${ }^{9}$ However, in such cases, more attention needs to be paid to the complications (such as infection and bleeding) associated with abruption of the membranes than to umbilical cord prolapse or placental abruption. A labor strategy should be decided based on frequent ultrasound examinations under full monitoring, labor management under a double setup, and the progress of labor.

In Western countries, double-balloon catheters are used for induction of labor. This case could have been avoided by using a double-balloon catheter. However, Salim et al. ${ }^{10}$ reported that, both, the single balloon and double-balloon catheters are equally effective for inducing labor and the double-balloon catheters may be associated with more operative deliveries. Pennell et al. ${ }^{11}$ reported that the single balloon catheter offers the best combination of safety and patient comfort.

Other mechanical methods for pre-induction cervical ripening were reported by Durie et al. ${ }^{2}$ They reported that none of the alternative methods had been shown to be superior to the Foley's catheter, while the mechanical methods such as osmotic dilators, extra-amniotic saline infusion, and double-balloon catheters have been shown to be safe and effective for cervical ripening.

While the need for insertion of an obstetrical single balloon by a skilled practitioner and confirmation of the position are essential, in the present case, we were keenly aware of the need for flexibility in our resourcefulness in an unexpected case, taking into account the potential complications such as infection and increased bleeding.

When using the single balloon catheter, a physician should be careful and watchful of its position. In case it aberrates into the uterine cavity, we suggest performing artificial rupture of the membranes in order to avoid placenta abruption.

\section{Acknowledgements}

The authors would like to thank Dr. Naveen Manohar, who provided medical writing services on behalf of Editage by CACTUS.

\section{Conflicts of interest}

The authors report no conflict of interest concerning the materials or methods used in this case report or the findings specified in this paper. The authors have no competing financial interests related to these case reports.

\section{References}

1. Mei-Dan E, Walfisch A, Valencia $\mathrm{C}$, et al. Making cervical ripening EASI: a prospective controlled comparison of single versus double balloon catheters. J Matern Fetal Neonatal Med. 2014;27(17):17651770 .

2. Durie D, Lawal A, Zegelbone P. Other mechanical methods for preinduction cervical ripening. Semin Perinatol. 2015;39(6):444-449.

3. Krammer J, O Brien WF. Mechanical methods of cervical ripening. Clin Obstet Gynecol. 1995;38(2):280-286.

4. Sherman DJ, Frenkel E, Tovbin J, et al. Ripening of the unfavorable cervix with extraamniotic catheter balloon: clinical experience and review. Obstet Gynecol Surv. 1996;51(10):621-627.

5. Hasegawa J, Ikeda T, Sekizawa A, et al. Obstetric risk factors for umbilical cord prolapse: a nationwide population-based study in Japan. Arch Gynecol Obstet. 2016;294(3):467-472. 
6. Yamada T, Cho K, Yamada T, et al. Labor induction by transcervical balloon catheter and cerebral palsy associated with umbilical cord prolapse. J Obstet Gynaecol Res. 2013;39(6):1159-1164.

7. Yamada T, Kataoka S, Takeda M, et al. Umbilical cord presentation after use of a trans-cervical balloon catheter. J Obstet Gynaecol Res. 2013;39(3):658-662.

8. Lind BK. Complications caused by extramembranous placement of intrauterine pressure catheters. Am J Obstet Gynecol. 1999;180(4):1034 1035 .
9. McMaster K, Sanchez-Ramos L, Kaunitz AM. Evaluation of a Transcervical Foley Catheter as a Source of Infection: A Systematic Review and Meta-analysis. Obstet Gynecol. 2015;126(3):539-551.

10. Salim R, Zafran N, Nachum Z, et al. Single-balloon compared with double-balloon catheters for induction of labor: a randomized controlled trial. Obstet Gynecol. 2011;118(1):79-86.

11. Pennell CE, Henderson JJ, O Neill MJ, et al. Induction of labour in nulliparous women with an unfavourable cervix: a randomised controlled trial comparing double and single balloon catheters and PGE2 gel. BJOG. 2009;116(11):1443-1452. 